\title{
Detection ischemic episodes from electrocardiogram signal using wavelet transform
}

\author{
Mohammad Karimi Moridani, Majid Pouladian
}

Biomedical Engineering Department, Science and Research Branch, Islamic Azad University, Tehran, Iran

Email: Karimi.m@srbiau.ac.ir

Received 19 November 2008; revised 16 March 2009; accepted 16 April 2009.

\begin{abstract}
In this paper, we propose an algorithm for detection of myocardial ischemic episodes from electrocardiogram (ECG) signal using the wavelet transform technique. The algorithm was tested on data from the European ST-T change database. Results show that this algorithm is effective for distinguishing normal ECGs from ischemic. We developed a method that uses wavelets for extracting ECG patterns that are characteristic for myocardial ischemia.
\end{abstract}

Keywords: Myocardial Ischemic; Wavelet Transform; ECG

\section{INTRODUCTION}

The formulation and properties of an electrical impulse through the heart muscle result in time-varying potentials on the surface of the human body, which are known as the ECG signals. The signal represents various activities of the heart [1]. Wavelet Transformation (WT) has shown to be substantially noise-proof in ECG segmentation, and thus very appropriate for ST-T segment extraction (Figure 1). An initial downward deflection after the $\mathrm{P}$-wave is termed as, 'Q', the dominant upward deflection is the ' $R$ ' and the terminal part is denoted as ' $S$ '. The T-wave represents ventricular recovery or repolarization $[1,2]$.

The ST segment usually merges smoothly and imperceptibly with the T-wave.

Having a simple estimate of whether an ECG recording which includes segments is characteristic for ischemic heart or not, is one of the most interesting topics for cardiologists.

Our aim in this research is to develop an algorithm using WT for identification of myocardial ischemic episodes.

\section{METHOD}

For ECG parameters estimation, it is desirable that the

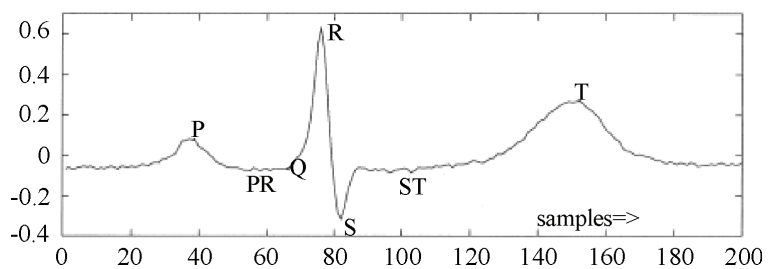

Figure 1. ECG waveform.

basis functions (wavelets), be symmetric/antisymmetric Asymmetric basis will enable to detect the extreme of wave's peak [4].

In case of antisymmetric basis, the peak of the wave is detected as a zero crossing.

In order to have a computationally simple analysis, the peaks should be detected as zero crossings (which is provided by antisymmetric basis) or local extreme (symmetric basis) [4]. It is also desirable that basis have a minimum number of sign changes. In practice, QRS complex is usually considered to be symmetrical, while $\mathrm{T}$ wave is less so. However, it has been shown, PR and ST points can be estimated using biorthogonal wavelets under the assumption of QRS complex and $\mathrm{T}$ wave symmetry [2]. Moreover, having as an aim proposed quantitative analysis, it is quite plausible to suppose basic QRS complex and T wave symmetry [4]. Biorthogonal wavelets satisfy both these properties, so they are used for DWT (discrete wavelet transform) decomposition. Hence used in this work. The filter coefficients of both the symmetric low pass (LP) $H$ and the antisymmetric high pass (HP) filters $G$ and $K$ are given in Table 1 [2]. Decomposition and reconstruction filters satisfy further equations:

$$
|H(w)|^{2}+G(w) K(w)=1
$$

where, $w$ is frequency

$$
\begin{aligned}
& H(w)=e^{i w 2}(\cos (w / 2))^{p} \\
& G(w)=4 i e^{i w 2} \sin (w / 2)
\end{aligned}
$$




$$
\begin{gathered}
K(w)=\frac{1-|H(w)|^{2}}{G(w)} \\
i=\sqrt{-1} \text { and } \mathrm{p}=3,5,7, \ldots
\end{gathered}
$$

For compact support ' $p$ ' should be as small as possible. Hence, it was chosen to be equal to 3 . The filter coefficients corresponding to $p=3$ are given in Table 1 .

\subsection{Parameters Estimation}

The parameters of the ECG signal are obtained by the wavelet decomposition dyadic tree. This tree decomposes the signal initially into the smooth (low pass) and detail (high pass) constituents [1]. The low pass component is further decomposed into low pass and high pass. This process is repeated over the desired number of scales. When an ECG signal is passed through each of the wavelet filters whose scales range from 21 to 24 , as shown in Figure 2, the detailed $d 1, k, d 2, k, d 3, k d 4, k$ and the smooth $s 1, k, s 2, k, s 3, k, s 4, k$ filtered outputs are obtained as shown in Figure 3. The following algorithm is suggested for estimating the said ECG parameters [1].

This initial filter is also based upon WT. The denoising procedure is performed in three steps (MATLAB wavelet toolbox) [2]:

1) decomposition with sym8 wavelet at level 5 ,

2) detailed coefficients threshold- choosing a threshold for each level,

3) reconstruction.

In order to obtain ST and PR points' values, each decomposed ECG is segmented (Figure 1). At the first and the second levels, QRS starting, ending and peak point are

Table 1. Filter coefficients of symmetric Low Pass (LP), antisymmetric High Pass (HP) and their reconstruction filters.

\begin{tabular}{cccc}
\hline $\mathrm{H}$ & $\mathrm{G}$ & $\mathrm{K}$ & $\mathrm{L}$ \\
\hline 0.1768 & 0.3536 & 0.1768 & -0.3536 \\
0.5303 & 1.0607 & -0.5303 & 1.0607 \\
0.5303 & -1.0607 & 0.5303 & 1.0607 \\
0.1768 & -0.3536 & -0.1768 & -0.3536 \\
\hline
\end{tabular}

G, decomposition high pass filter coefficients; H, decomposition low pass filter coefficients; K, reconstruction high pass filter coefficients; L, reconstruction low pass filter coefficients.

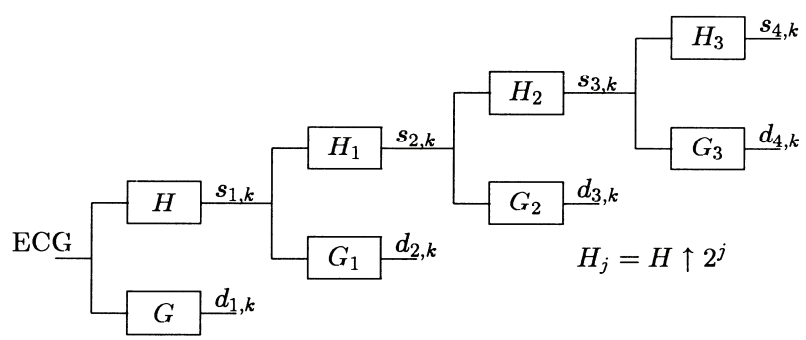

Figure 2. Wavelet decomposition of ECG signal. Decomposition per-formed over 4 scales. extracted. Let us denote as $n_{R}$-the $\mathrm{R}$ peak point, $\mathrm{nl}$, and $\mathrm{n}, 2$ the beginning and ending points of QRS complex respectively [2]. PR point is thus calculated as:

$$
\text { PRpoint }=n_{R}-\frac{n_{l 2}-n_{l 1}}{2}
$$

At the fourth level, ST-T characteristic points are obtained: $n_{T}$ - the T peak point, nt 1 and $n t 2$ the beginning and ending points of $\mathrm{T}$ wave, respectively [1]. ST point is calculated as:

$$
\text { STpoint }=n_{T}-\frac{n_{l 2}-n_{l 1}}{2}
$$

Wavelet decomposition introduces scale-dependent phase delay into signals. For example, each zero crossing point which corresponds to the peak of a symmetric uniphase wave is delayed for exactly $2^{J-1}-1$ points, where represents the scale [1].

\subsection{ST Deviation Analysis}

In our analysis, we have used European ST-T change database with ischemic ECG signals sampled at $250 \mathrm{~Hz}$, two hours in duration each. The most elementary differentiate/threshold algorithm has been applied to extract each beat. Obtained signals were decomposed as described in section 2.1 and for each beat/separate signal, an ST deviation value was calculated as [2]:

$$
\begin{aligned}
& \text { STdeviation = } \\
& \mid \text { ECGvalue (PRpo int) - ECGvalue (STpo int) }
\end{aligned}
$$

where ECG value(x) denotes an amplitude at the point $\mathrm{x}$ of a given ECG.

Cases with specific ECG beats, that is to say with indistinguishable ECG features (thus impossible segmentation), were excluded from further analysis. In this way, an ST deviation value was obtained for each beat that could be analyzed [2]. The final report might include the comment that some beats were excluded from analysis as well as the number of the beats omitted, and therefore suggest a manual observation where required [2].

\subsection{QRS Onset and Offset Detection}

Some of the existing techniques use a series of bandpass filters to extract $Q R S$ complexes from the ECG signal, which under severe baseline drift and other high frequency noises, fails to detect the characteristic points to an acceptable accuracy. And some techniques use neural network based adaptive identification algorithms $[3,5]$, which can be used for only a particular type of pattern. The wavelet transform based technique can be used to identify the characteristic points of the ECG signal to a fairly good accuracy, even with the presence of severe high frequency and low frequency noises $[6,7,8]$. Our aim is to describe an elegant algorithm, which uses 
WT to identify the characteristic points of the ECG signal, and identifying the myocardial ischemic episodes.

As an alternative to the normal filtering techniques, which use different narrow-band filters to extract the frequency contents of the signal, wavelet transform technique can be used [3]. In wavelet transform technique, the signal is analyzed at different frequencies with different resolutions. It is called multi resolution analysis (MRA) (Figure 4).

The wavelet used in this work is the quadratic spline wavelet [9]. The reasons for choosing this particular wavelet for the analysis purpose are as follows:

It has a very compact support and a generalized linear phase, so there is a determinate relationship between ECG characteristic points and the modulus maxima, or the zero-crossing points of the WTs.

The Fourier transform (FT) of the quadratic spline wavelet is given as:

$$
\varphi(w)=i w\left(\frac{\sin \frac{w}{4}}{\frac{w}{4}}\right)^{4}
$$

The FIR(Finite Impulse Response) filter coefficients that make up the decomposition and reconstruction filter banks and the Lipschitz coefficients for the decomposition algorithm are given in Tables 2 and 3 [3].

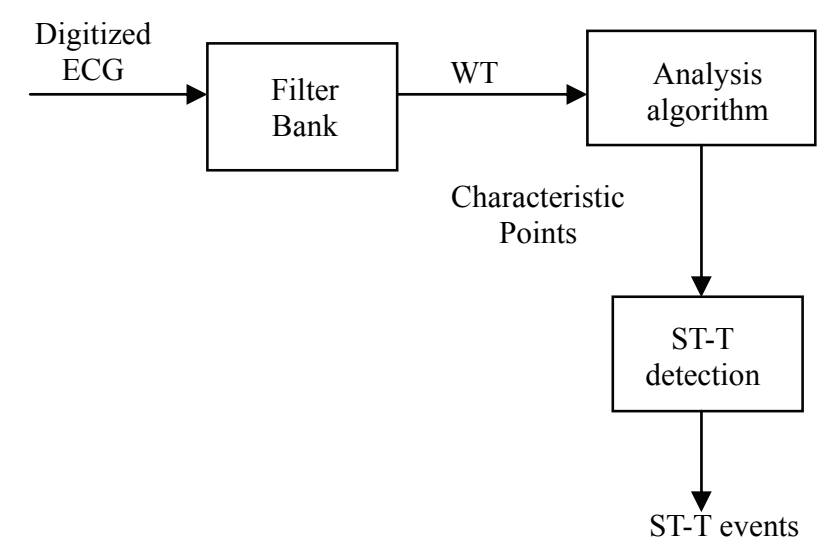

Figure 4. Block diagram of the entire process.

Table 2. FIR filter coefficients for quadratic spline wavelet.

\begin{tabular}{cccll}
\hline$N$ & $H$ & $G$ & \multicolumn{1}{c}{$K$} & \multicolumn{1}{c}{$L$} \\
\hline-3 & & & 0.0078125 & 0.0078125 \\
-2 & & & 0.054685 & 0.046875 \\
-1 & 0.125 & & 0171875 & 0.1171875 \\
0 & 0.375 & -2.0 & -0.171875 & 0.65625 \\
1 & 0.375 & 2.0 & -0.054685 & 0.1171875 \\
2 & 0.125 & & -0.0078125 & 0.046875 \\
3 & & & & 0.0078125
\end{tabular}

G, decomposition High Pass filter coefficient; H, decomposition Low Pass filter coefficients; K, reconstruction High Pass filter coefficients; L, reconstruction Low Pass filter coefficients.
Table 3. Normalization coefficients $\lambda_{j}$ for the quadratic spline wavelet.

\begin{tabular}{lc}
\hline$j$ & $\lambda_{j}$ \\
\hline 1 & 1.5 \\
2 & 1.12 \\
3 & 1.03 \\
4 & $1 . .01$ \\
5 & 1.00 \\
\hline
\end{tabular}

There is a relation between the characteristic points of the signal and their WT at different levels $[10,11,12]$. For example, for the wave in Figure 5, the wavelet transform at scale $2^{1}$ is given.

The wave's rising edge corresponds to a negative minima and the dropping edge corresponds to a positive maxima. The moduli of these maxima or minima corresponding to the same edge are named as the modulus maxima line. If the uniphase wave is symmetric to its peak, then its peak corresponds to the zero-crossing point of the positive maxima negative minima pair with a delay of exactly $2^{J-1}-1$ points, where $j$ represents the scale [3]. After obtaining the wavelet transform coefficients at different scales, the next step is to find out the ECG characteristic points from these coefficients [3].

\subsection{T and $P$ Wave Detection}

$T$ and $P$ waves are normally low frequency, so WT at high scale is used to locate these waves. In this work, WT up to four scales are taken and the scale $2^{4}$ is used to locate $T$ and $P$ waves.

$T$ wave creates a pair of modulus maxima with a different sign on $W 2 j f(n)$ at scale, $2^{4}$ within a time window after the detected $R$-peak [3]. Since the wave is almost symmetric to its peak, the peak of $T$-wave corresponds to the zero crossing point of the modulus maximum pair with a delay of $2^{4-1}-1$ points. The peak, onset and offset of the $P$-wave are detected in a way similar to those of the $T$ before the detected $R$-wave [3].

\subsection{R-peak Detection}

For detecting the $R$-peak, the modulus maxima-minima pair is located at lowest scale, which is done by fixing a threshold for detection [13]. So maxima-minima pairs for other scales are located within the neighborhood of these maxima-minima pairs. If the amplitudes of the maxima-minima pairs, compared to those are at lower scale, are consistent or increasing, the corresponding

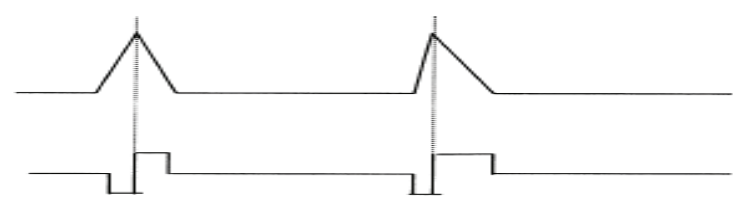

Figure 5. The uniphase wave and its WT at scale $2^{1}$. 
modulus maxima-minima pair is treated as one that corresponds to a true $R$-peak [3]. This method reduces the effect of low frequency artifacts and also the high frequency non morphological noise.

\subsection{Detection of MI from the ECG Characteristic Point}

Different ECG changes related to the evolution of ischemia have been described, including $T$-wave amplitude changes, $S T$ deviations and even alterations in the terminal portion of the $Q R S$ complex [13]. Using global representations for the $S T-T$ complex instead of a single point from the $S T$ segment could better characterizes ischemia patterns and yield better identification of occluded artery [14,15]. The most important ECG change associated with ischemia is the $S T$ segment elevation or depression, with depression being most common. Also, this can be along with $T$-wave amplitude changes or even inversion [3]. So ischemia can be detected using these two measurements. For finding the $S T$ depression level, a reference level is found out at first. This is done by drawing a line between two or more $P$-waves where they return to the base line (or starting of $P$-wave). From the characteristic point detection algorithm, we obtain the $P$-wave onset and offset of all the cardiac cycles. $S T$-segment is the segment of ECG between $Q R S$ offset and $T$-wave onset. The deviation of this segment from the reference line is found out. The amplitude of $T$-wave is also found by measuring the distance between $T$-peak and reference line [3]. Having obtained these two values, we can come to a conclusion, that whether the cardiac cycle contains an ischemic episode or not.

\section{INTERPRETING THE DATA}

Two or more ST change episodes could be separated by insignificant time gaps or with an unreadable ECG segment. This could lead to the conclusion that these episodes are separate, which is scarcely the case. Since the duration of the episodes are of great importance for making conclusions about myocardial ischemia, it was necessary to alleviate such shortcomings [2]. Thus we applied a low pass filter (Chebyshev Type I filter) to the latter signal; thereby, constructed an envelope which was informative enough [2].

Figure 6 shows typical results from the readings in a patient with myocardial ischemia (manifested through T-and ST-wave change) [2]. High values at vertical axis suggest that a long ischemic episode has occurred (A part, Figure 6). Within normal ECGs, we expect that these values gather near zero. B part at Figure 6 is a direct consequence of final low pass filtering and suggests that a series of shorter, but still significant, ST episodes have occurred with unsubstantial time gaps among them. B parts are not expected with normal ECGs.
Negative ordinate values are a co product of LP filtering and bare no practical significance [2]. Beside ECG analyzed in Figure 6 which we considered to be a mid-case in the terms of number of ST deviations, we selected two more examples in order to demonstrate the efficiency of the algorithm.

Figure 7 presents performance of the algorithm in the case where there were only few ST changes, while Figure 8.

In Figure 7, we can clearly see that vertical axis values are smaller, compared to those in Figure 6 or Figure 8. Moreover, there are less high-valued ripples, and they occur with significant time gaps. This suggests that analyzed ECG is near normal in the terms of ST change [2].

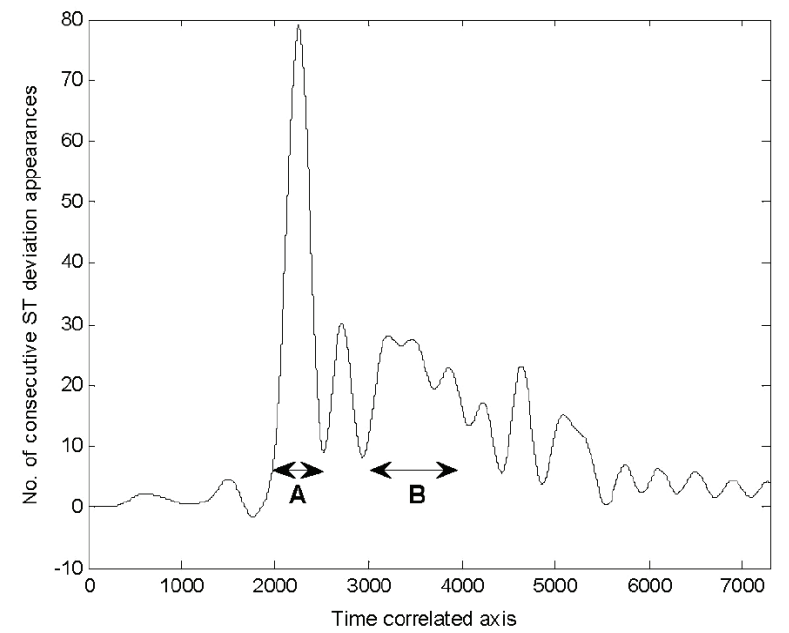

Figure 6. Number of ST deviations correlated with time of consecutive appearances-function's envelope.

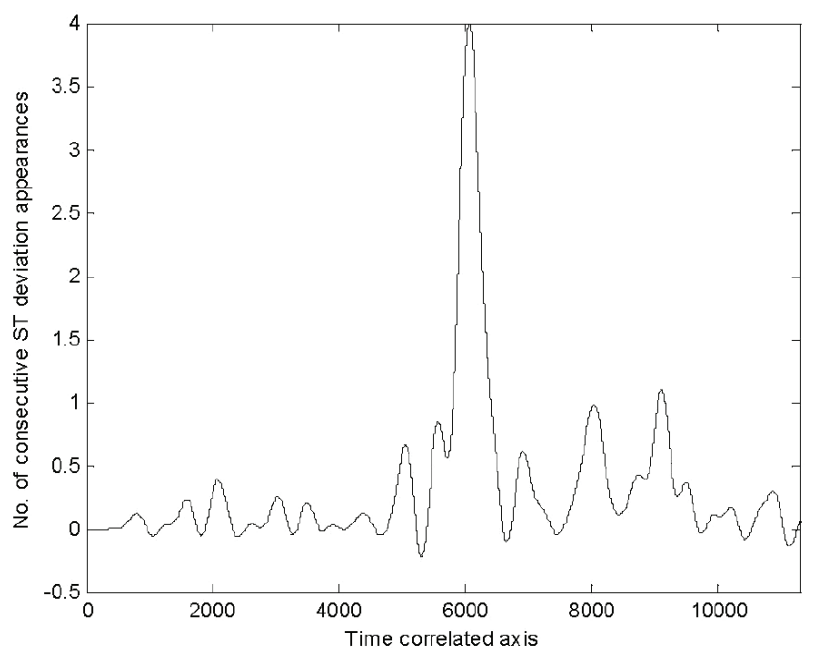

Figure 7. Number of ST deviations correlated with time of consecutive appearances-case with few ST-T deviations. (file e115. dat in has been analyzed) [4]. 


\subsection{Estimating Algorithm's Efficiency}

Annotation files provided for ST-T change ECG signals in contain information about ST and T-wave deviations as well as the number of heart beat in that particular ECG at which a change occurred [4]. ST changes are annotated by values ' 18 ' and T-wave changes by ' 19 '.

In order to demonstrate similarity which exists between the algorithm's outcome and manually annotated ST-T changes, in Figure 6 and Figure 7 we present the manually annotated change/time dependence for the same ECGs analyzed in Figure 7 and Figure 8, respectively [2].

Comparing Figure 7 and Figure 9, as well as Figure 8 and Figure 10, it shows the strong correlation between automatic and manual ECG analysis. Direct relationship exists between the time axes, too, but the algorithm's outcome 'compresses' time axis due to the nature of the constructed array whose envelope is the actual final result [2].

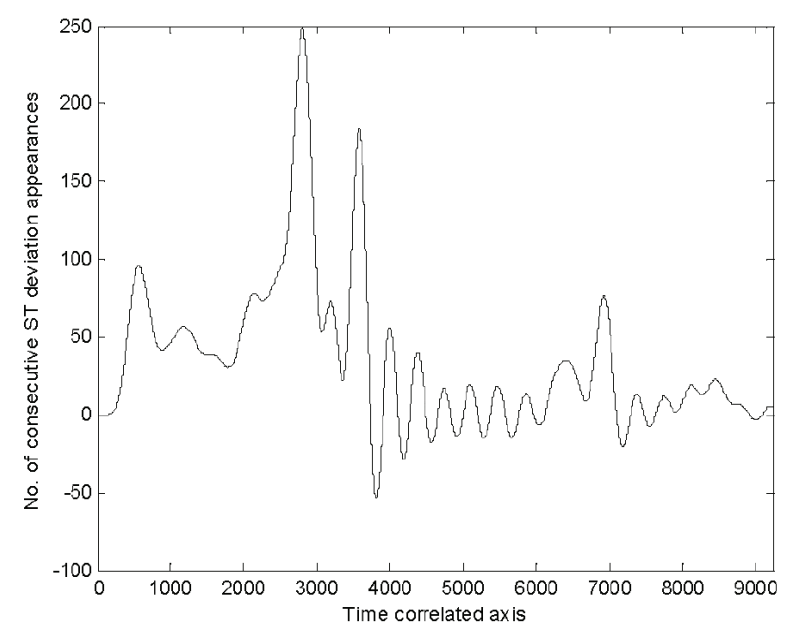

Figure 8. Number of ST deviations correlated with time of consecutive appearances-case with a considerable number of ST-T deviations. (file E121.dat in has been analyzed) [4].

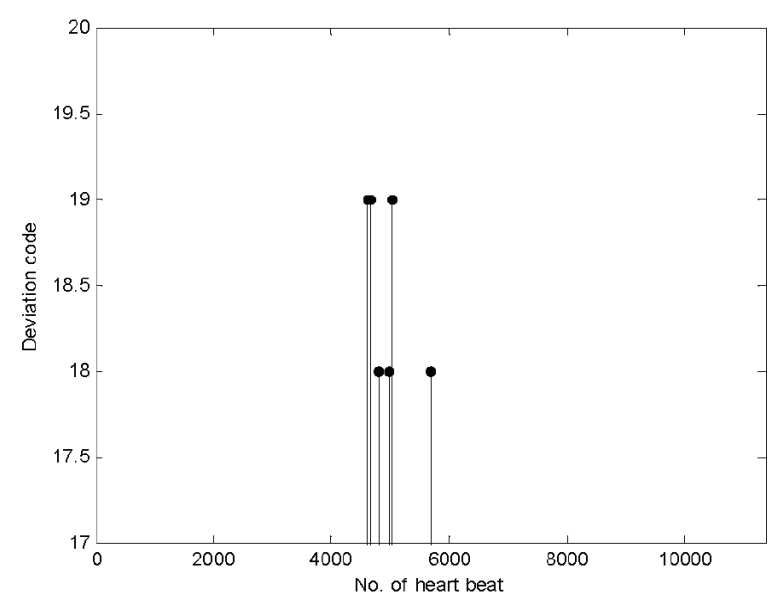

Figure 9. Manually annotated ST-T changes which correspond to Figure 7. (file e115.atr in has been used) [4].

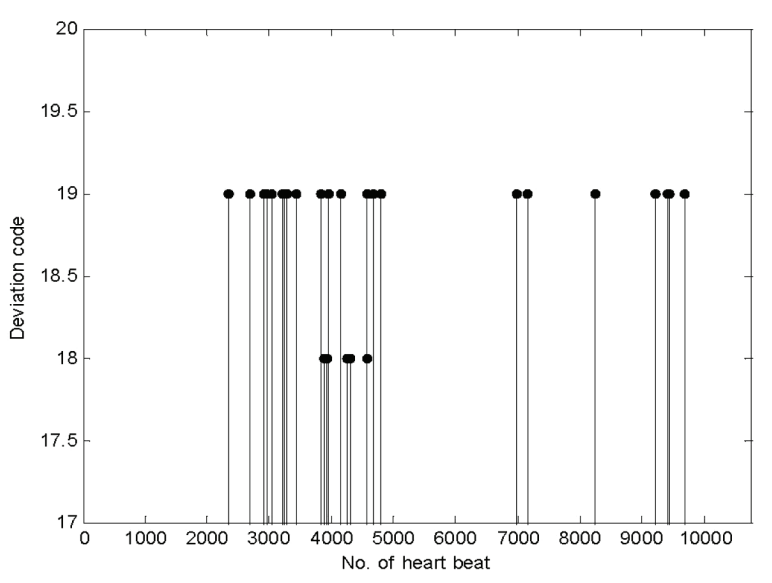

Figure 10. Manually annotated ST-T changes which correspond to Figure 4. (file e115.atr in has been used) [4].

After such analysis, a physician can examine those parts of ECG which were annotated for ST changes, if necessary. Performance on annotating ST changes of the proposed algorithm is the same as of, although different wavelet base function had been used [2]. Demonstrated performance has been verified on 10 ECGs from, and confirms already described relationship between ECGs automatically and manually analyzed for the stated purpose [4].

\section{RESULT}

An array of ST deviations was transformed into an array which correlates number of ST deviations with time of consecutive appearances [2] to show that the algorithm counts the number of consecutive appearances of ST deviations greater than $0.1 \mathrm{mV}$ and uses that number as a new array's element. The procedure is repeated for each ST change episode.

$S T-T$ change detection algorithm consists of calculating certain performance indices. They are: $S T$ sensitivity $\left(S T_{\mathrm{se}}\right)$, which is an estimation of the likelihood between detecting an ischemic $S T$ episode; $S T$ positive predictivity $(S T+P)$, an estimation of the likelihood that a detection is a true ischemic $S T$ episode; ischemic sensitivity $\left(I S_{\text {se }}\right)$, which is a fraction of true ischemia, and ischemic positive predictivity $(I S+P)$, which is the fraction of detector annotated ischemia, and is true ischemia $[3,16]$.

\section{CONCLUSIONS}

In this paper, we proposed an algorithm for the detection of myocardial ischemic episodes from electrocardiogram (ECG) signal using the wavelet transform technique.

The study is mainly aimed to use biorthogonal wavelets to estimate clinically significant parameters of ECG waveforms and find out ST-T segment for detection ischemic episodes. Comparing the measured ST and the normal ST in test ECG provided by European ST data- 
base, the algorithm could found the exactly time, that ST shape is changed into an abnormal shape. ST segment is the most important diagnostic parameter to finding myocardial ischemia therefore developed algorithm best coincidence with the database occurs in the determination of the beginning of the ST episodes, and the worst in the calculation of the maximum deviation. Nevertheless, this parameter is just used in the description of ischemia episodes, and its importance is relatively low with respect to the quantification of the number of episodes and their duration.

\section{REFERENCES}

[1] N. Sivannarayana, D. C. Reddy, (1999) Biorthogonal wavelet transforms for ECG parameters estimation, 167-174.

[2] N. Milosavljevic and A. Petrovic, (2006) ST segment change detection by means of wavelets, 137-140.

[3] P. Ranjith, P. C. Baby, and P. Joseph, (2003) ECG analysis using wavelet transform: application to myocardial ischemia detection, 44-47.

[4] "European ST-T change database," www. physionet.org.

[5] N. Magaveras, T. Stamkopoulos, C. Pappas, and M. G. Strintzis, (1998) An adaptive backpropagation neural network for real-time ischemia episodes detection: Development and performance analysis using the European ST-T database, IEEE Trans Biomed Eng, 45, 805-13.

[6] M. Vetterly and J. Kovacevic, (1995) Wavelet and sub- band coding, First Edition, Upper Saddle River, NJ: Prentice Hall.

[7] M. R. Raghuveer and S. Bopardikar, (1996) Wavelet transforms: Introduction to theory and applications, Second Edition, Boston, MA: Addison Wesley.

[8] (1996) Special issue on wavelets, Proc IEEE, 84(4).

[9] S. Mallat and S. Zhong, (1992) Characterization of signals from multiscale edges, IEEE Trans PAMI, 14(7).

[10] C. Li, C. Zheng, and C. Tai, (1995) Detection of ECG characteristic points using wavelet transform, IEEE Trans Biomed Eng, 42(1), 8-21.

[11] D. P. Golden, R. A. Wolthuis, and G. W. Hoffler, (1973) A spectral analysis of the normal resting electrocardiogram, IEEE Trans Biomed Eng, 20(5), 366-72.

[12] S. Mallat, (1991) Zero crossing of a wavelet transform, IEEE Trans Inf Theory, 37(4).

[13] P. F. Cohn, (1993) Silent myocardial ischemia and infarction, 8-18, NewYork: Marcel Dekker, 87-97.

[14] F. Jager, G. B. Moody, and R. G. Mark, (1998) Detection of transient ST segment episodes during ambulatory ECG monitoring, Comput Biomed Res, 31(5), 305-22.

[15] J. Garcia, L Sornmo, S. Olmos, P. Laguna, (2000) Automatic detection of ST-T complex changes on the ECG using filtered RMS difference series: Application to ambulatory ischemia monitoring, IEEE Trans Biomed Eng; 47(9), 1195-201.

[16] F. Jager, G. B. Moody, A. Taddei, R. G. Mark, (1991) Performance measures for algorithms to detect transient ischemic ST segment changes. Computers in Cardiology, Los Alamitos, CA: IEEE Computer Society Press, 372-96. 